\title{
El gusto como un factor determinante en el ejercicio crítico del diseño*
}

Recibido: 2015-04-09 -Enviado a pares: 2015-04-15

Aprobado por pares: 2015-05-10 Aceptado: 2015-05-15

Juan José Cadavid Ochoa*

\begin{abstract}
Resumen
A partir de algunas reflexiones gadamerianas y de otras discusiones contemporáneas en torno a la idea de juicio estético, se propone entender el papel del gusto en el ejercicio valorativo en los procesos evaluativos y, por lo tanto, críticos de las disciplinas del diseño, entendidas como un saber-hacer artístico y comunicacional.
\end{abstract}

Palabras clave: diseño, gusto, juicio, subjetivo, método,

* Este artículo es resultado del proyecto de investigación (en curso) titulado Currículo transmedial, una alternativa para la formación en artes, a cargo del Grupo de Investigación Código. (Colombia)

** Magíster en Estudios Humanísticos, candidato a Doctor en Humanidades en la Universidad EAFIT y docente en la Institución Universitaria Salazar Herrera (e-mail: j.cadavid@iush.edu.co). 


\title{
Taste as a determinant factor in critically exercising design
}

\begin{abstract}
Based on several Gadamerian reflections and other contemporary discussions on the aesthetic judgment idea, this article intends to achieve an understanding of taste in the value-assessing exercise of the evaluative and, therefore, critical processes of the design disciplines, understood as an artistic and communicational know-how.
\end{abstract}

Key words: design, taste, judgment, subjective, method.

\section{Gosto como um fator crítico para o exercício de design}

\section{Resumo}

A partir de alguns gadamerianas reflexões e outras discussões contemporâneas em torno da idéia de juízo estético, propõe-se a compreender o papel de gosto no exercício de avaliação em processos de avaliação e disciplinas de design, portanto, críticas entendida como um perícia artístico e comunicacional.

Palavras-chave: design, gusto, julgamento, subjetiva, método, 


\section{Introducción}

Este artículo es resultado parcial de la investigación en curso: Currículo transmedial, una alternativa para la formación en artes. Aquí, se realiza una reflexión sobre el concepto del gusto, en relación con las metodologías de evaluación del ejercicio del arte y el diseño, partiendo de las propuestas que, sobre tal idea, realiza el filósofo Gadamer, H. en Verdad y método (1960). De estas últimas se procura construir un marco referencial y conceptual sobre el cual se soporte una construcción pedagógico-didáctica, específica para la educación artística.

\section{Metodología}

Para lograr esto se realiza una lectura hermenéutica de diferentes textos de referencia, procurando establecer un distanciamiento conceptual con algunas concepciones modernas y racionalistas cercanas a propuestas positivistas, que pretenden definir las disciplinas artísticas como prácticas dependientes de métodos en los que el gusto carece de potencia objetiva y debe ser normativizado. Para construir así una propuesta con pretensiones cientificistas -premisas racionales desde las cuales sólo lo observable es verdadero (Círculo de Viena, 1929)-, se utilizan criterios teóricos propios de la disciplina de la estética, que permitan discutir los estatutos que tradicionalmente se le han asignado, en tanto tendencias y procesos, así como dar cuenta de los límites y alcances del juicio del gusto en lo que respecta a sus aportes en el campo pedagógico y formativo del saber del diseño y las artes.

\section{El devenir del gusto, la historia del olvido de un concepto}

El interés por abordar la lectura hermenéutica de la estética para generar un juicio del gusto se debe tanto a la escasa producción desde el ámbito de la disciplina del diseño como a su poca recepción crítica y reflexiva por parte del mundo académico e intelectual. Esta reflexión pretende explicar cuál es el papel y la importancia del gusto como factor determinante en el arte y el diseño y, por lo tanto, como soporte crítico estético de conocimiento artístico. Para ello se puede afirmar, en principio, y como presupuesto fundamental, que el gusto es un concepto que implica un juicio.

Originalmente el concepto gusto proviene del verbo de raíz latina: sapere, que significó poner en práctica el sentido del gusto y por lo tanto tener juicio, o tener la capacidad de comprender y diferenciar lo bueno de lo malo en las vivencias de los hombres. Tal capacidad convierte a la humanidad en una especie con sapiencia, es decir, que a través del gusto las personas tienen la facultad de conocer. El gusto, en un sentido estético, ha sido un concepto importante frente al cual se ha reflexionado en diferentes épocas; el pensamiento alrededor del gusto se ha ocupado de la relación emotiva y racional de los individuos frente a una experiencia, en tanto esta produce un saber y un sabor, es decir, permite experimentar grados de satisfacción con o sin interés.

En este sentido, es importante realizar un sobrevuelo histórico sobre los planteamientos centrales del gusto estético que se ubican a finales del siglo XVIII y principios del 
XIX. En estos períodos algunos pensadores se preguntaron si el gusto era una facultad o una capacidad, es decir, si el gusto era connatural al ser humano o más bien dependía de condiciones culturales; al mismo tiempo, se cuestionaba el carácter racional o la condición sensible de tal concepto; así, importantes reflexiones como: Sobre la norma del gusto, de Hume (1757) en el que escribió: "es natural el que busquemos una norma del gusto, (a) una regla con la cual puedan ser reconciliados los diversos sentimientos de los hombres, (b) o al menos una decisión que confirme un sentimiento y condene el otro" (Hume, 1989, p. 27). También fueron importantes las reflexiones escritas en: El ensayo sobre el gusto, de Gerard (1759/1992), La crítica del juicio, de Kant (1790/1995), Los placeres de la imaginación, de Addison (1991), La investigación sobre el origen de nuestras ideas de belleza y de virtud, de Francis Hutcheson (1992). Estos y otros trabajos teóricos estructuraron un sustrato filosófico y estético, que permite entender el fenómeno del gusto (juicio) en términos modernos y por lo tanto desde fundamentos objetivos que categorizan la belleza y la fealdad en el arte y en la naturaleza como extremos de los que se ocupaba la estética, pero permiten, al mismo tiempo, dilucidar una ruta hacia planteamientos de orden subjetivo; en tal sentido, se puede sintetizar diciendo que el gusto para los empiristas está definido por las sensaciones y los sentimientos que despierta en un individuo una vivencia o experiencia estética; de esa forma se realiza para estos teóricos una separación entre las características objetivas de lo bello y la noción subjetiva que depende de apreciaciones del gusto. Los planteamientos filosóficos alrededor del juicio estético en el siglo XVIII son contenidos por Schiller en: Über die ästhetische Erziehung des Menschen (Sobre la educación estética del hombre) y Über naive und sentimentalische Dichtung (Sobre la poesía ingenua y sentimental) entre 1785 y 1795.

En el siglo XIX el interés por la reflexión estética decae, y en el XX el pensamiento de Gadamer, y en especial su trabajo estético contenido en La actualidad de lo bello y en Verdad y método, redimensiona en términos hermenéuticos la discusión sobre el gusto, poniendo la condición subjetiva del juicio estético en una estrecha relación con las condiciones sociales, políticas, económicas, es decir, con las construcciones antropológicas colectivas, particulares y generales de la condición humana. El pensamiento hermenéutico supera y salda la dicotomía objetividad-subjetividad al plantear una necesidad analítica relacional en la que los individuos construyen su capacidad de juicio en relación con fenómenos culturales externos a ellos mismos y siempre en condiciones sociales, es decir, de manera intersubjetiva.

Es a partir de estas reflexiones gadamerianas y de otras discusiones contemporáneas, que se propone entender el papel del gusto en el ejercicio valorativo en los procesos evaluativos y, por lo tanto, críticos de las disciplinas del arte y del diseño actuales. Para lograrlo hay que desprenderse de la concepción moderna y racionalista que pretende definir el diseño como una práctica anclada y dependiente de un método en el que el gusto -un concepto con una carga altamente subjetiva-carece de potencia objetiva y debe, como dijo Hume, ser normativizado, para construir así una propuesta con pretensiones cientificistas; esto implica que se debe interrogar y argumentar cómo el concepto del gusto afecta distintos momentos procesuales de algunas de las estructuras proyectuales herederas del discurso positivista. 


\section{Hacia una hermenéutica del gusto}

En torno al gusto hay que entender que este es un fenómeno estrechamente relacionado con la moda, pues esta es la plataforma social que contiene y generaliza al concepto del gusto, es decir, ya no como una condición individual extraída de cualquier influencia contextual, sino todo lo contrario: como el resultado de ciertas conexiones y acuerdos sociales dominantes. Pero paradójicamente la moda -que según la real academia de la lengua española ${ }^{1}$ puede definirse como: el uso o la costumbre que está en boga durante algún tiempo, o en determinado país- implica un modo (modus) o en francés mode, es decir, una forma particular y dinámica de hacer las cosas, una manera cambiante insertada en el todo permanente del comportamiento social. La sociedad generaliza el concepto de gusto, instalando un acuerdo, un convenio, un modo dominante que se hace moda y continuamente es reemplazado.

La moda entonces se caracteriza principalmente por imponer como norma aquello que todo el mundo hace en un período de tiempo y en un lugar determinado, regulando de manera caprichosa y aleatoria cosas, formas de vida y comportamientos que igual podrían ser de muchas otras formas; esto, porque los grupos humanos pueden tener diferentes formas de pensar, determinadas por las situaciones vividas y la pertenencia a grupos desde donde se construyen sistemas de valores no solo distintos, sino también cambiantes.

Tales visiones del mundo o cosmovisiones determinan modas, que constituyen por sí mismas una generalidad empírica; en tal sentido se crea una dependencia social a la que es difícil sustraerse, para Kant éste es el punto esencial, aunque sobre la moda considera que es "mejor ser un loco en la moda que contra la moda, aunque por supuesto sea también una locura tomarse las cosas de la moda demasiado en serio² (Gadamer, 2000, p. 69).

Puede decirse, siguiendo a Gadamer, que el gusto condiciona las modas, las determina, pero no se somete a ellas, ya que es por sí solo una capacidad de discernimiento sensible, espiritual, y en este sentido es posible decir que el buen gusto es aquel que se acopla o encaja con la línea que propone cada moda, adaptándose a sus variaciones o, de manera contraria, es aquel que adapta las exigencias de la moda a un criterio propio, de tal forma que mantiene cierta distancia con el fenómeno de la moda o la asume de manera mesurada, sin seguir las imposiciones de las tendencias y creando un propio estilo,

1 moda. F. 1. Uso, modo o costumbre que está en boga durante algún tiempo, o en determinado país, de manera especial en los trajes, telas y adornos, sobre todo los recién introducidos. || 2. Conjunto de la vestimenta y los adornos de moda. || 3. Estad. Valor que aparece con mayor frecuencia en una serie de medidas. I| de $\sim$. LOC. ADJ. Que en un momento determinado goza de destacada aceptación. U. t. c. loc. adv. || pasar, o pasarse, algo de $\sim$. LOCS. VERBS. Perder actualidad o vigencia. || V. día de $\sim$, tienda de $\sim$ s.

modo. 1. 'Manera'. Cuando va seguido de una oración adjetiva, esta puede ser introducida por en (el) que o como: «Echo de menos el modo en el que yo vivía» (Tiempo [Esp.] 29.1.90); «Era un hombre corpulento, a juzgar por el modo como había distendido el somier» (Azancot Amores [Esp. 1980]).

2 Gadamer citando a Kant (Anthropologie in pragmatischer Hinsicht) en Verdad y método. 
esto es, conservando un punto de vista que estructura un gusto propio el cual adopta aquellas propuestas que caben en él, moldeándolas en su molde. En este sentido la moda es una construcción empírica, es decir, que surge de la experiencia social, pues tiene un sentido comunitario en el que se vuelven generales los juicios particulares, mientras que el gusto no pretende hacer coincidir juicios y, por lo tanto, no podrá existir un desacuerdo con él, pues el gusto permite oposición y riña, pero no implica generalidad. Kant en Kritik der Urteilskraft dice que ante las imposiciones tiránicas de la moda, el gusto permite conservar la libertad. "En ello estriba la fuerza normativa que le es propia, en que se sabe seguro del asentimiento de una comunidad ideal. La idealidad del buen gusto afirma así su valor en oposición a la regulación del gusto por la moda" (Gadamer citando a Kant, 2000, p. 70).

La importancia y la supremacía del gusto sobre los fenómenos de la moda radican en que este construye una manera propia de conocer el mundo, de interpretarlo y de reconstruirlo. Pero no de manera arbitraria o sin referente alguno; es más bien la capacidad de juicio reflexiva que constituye una manera de apropiación particular e individual de lo general, es decir, con referencia a un todo, de tal forma que sea la manera adecuada en un contexto determinado.

El gusto siempre está en relación con un todo, pero este todo no implica un objetivo o una finalidad, simplemente actúa como marco referencial frente al cual se tiene cierto sentido o inclinación; desborda los límites del juicio de lo que es bello tanto en la naturaleza como en el arte, y de la condición decorativa de las cosas; abarca el amplio ámbito de las experiencias, las costumbres y las convivencias; constituye y determina estilos de vida. El gusto procura de esta forma modos referenciados y diferenciados de relacionarse con el mundo y, por lo tanto, permite que el ejercicio creativo pueda insertarse de manera efectiva o no, en una esfera o ámbito social determinado.

El ejercicio del diseño es intersubjetivo, construye miradas diversas del mundo, propone nuevas maneras de relacionarse y de ser; por eso está siempre presto al cambio y es susceptible de interpretarse en múltiples sentidos, dependiendo del contexto en el que aparece. "La transformación de los instrumentos y de los productos de la actividad artística precede y condiciona necesariamente la transformación de los instrumentos de percepción artística, transformación lenta y laboriosa ya que conlleva desarraigar un tipo de competencia artística para sustituirlo por otro" (Bourdieu, 2010, p.82). Que su carácter sea intersubjetivo implica que el concepto del gusto tiene injerencia no solo en el ejercicio crítico, sino también en el creativo, y es uno de los factores que influyen en los proyectos estéticos, es decir, en aquellas construcciones humanas que propician y permiten tener experiencias sensibles. Este es el factor bajo el cual subyacen los cánones estéticos que son definidos en una sociedad por un grupo de personas, evidenciando los criterios, la información y el conocimiento que posee el diseñador para crear propuestas seductoras y exitosas. El gusto es, de acuerdo con lo expuesto, un factor que puede ser valorado y a partir de él se abre un campo de estudio y crítica del arte y el diseño, pues es un componente ineludible de su saber-hacer. 
Volviendo a las definiciones de gusto, en la Real Academia de la lengua española ${ }^{3}$ se dice que gusto es el Placer o deleite que se experimenta con algún motivo, o se recibe de cualquier cosa. Por lo tanto el nivel de placer provocado y experimentado en la contemplación de una propuesta de diseño podría ser evaluado. Otra acepción del concepto gusto dice que es la Facultad de sentir o apreciar lo bello o lo feo. Tal afirmación implica determinar qué es bello y qué es feo, por lo que tal facultad tendrá que ser analizada contextualmente, pues tanto la belleza como la fealdad dependen de múltiples factores que varían en cada cultura, en cada región geográfica, en el tiempo y en las sociedades en las que se inscriben tales juicios estéticos. Como menciona Baudelaire (1964) "la belleza es de las circunstancias y el boceto de las costumbres". Esto nos hace volver sobre la reflexión acerca de la moda, pues son los grupos humanos, cada vez más influenciados por las dinámicas del mercado, quienes al conformar comunidades llegan a acuerdos y definen para ellos qué es bello y qué no lo es. Hoy tales grupos se insertan en la sociedad del espectáculo ${ }^{4}$; esto implica que el concepto del gusto en la actualidad ya no solo depende de modas que son fruto de convenciones creadas por sociedades y micro-sociedades, sino que tales maneras estéticas de juzgar el mundo son impuestas por grandes multinacionales que procuran dirigir el gusto general de acuerdo con sus intereses económicos. Asistimos en este escenario a la desvalorización del carácter individual del gusto, a su masificación y, por lo tanto, a la inversión jerárquica en la que la moda dirige al gusto, creando de manera paradójica un escenario global de mal gusto, o por lo menos de un gusto estandarizado y desechable. Pero también al surgimiento de propuestas subversivas de todo tipo que procuran la creación de relaciones estéticas diferentes a las producidas en serie, propuestas que son capaces de leer contextos, culturas y diferencias, que logran que la oposición constituya una forma de construcción de estilos y definición marginal de nuevos gustos. Hoy, aunque se hable de aldea global, las comunicaciones sean masivas y la información del planeta esté al alcance de todos, es posible seguir reconociendo las diferencias y desde estas crear asociaciones, colectividades que constituyen subculturas fundadas en lo particular, en lo menor, como diría Lumman, grupos sociales que generan maneras, formas diferentes de comportamiento y de percepción del mundo, modus que pueden identificarse y entenderse como estilos y que son susceptibles de crítica y evaluación desde el entendimiento de las condiciones frente a las que se revelan y aquellas que retoman como fuente y sustrato de sus propuestas.

\section{La metodología proyectual y la injerencia del gusto}

En los procesos de creación artística y de diseño es posible identificar etapas y operaciones, pasos que determinan, a su vez los criterios de evaluación del proyecto. Los juicios de efectividad, eficacia, pertinencia, alcance, impacto y funcionalidad, entre otros, guardan una estrecha relación con la estructura de lo que se ha llamado en diferentes áreas,

3 Significados tomados del diccionario de la Real Academia De La Lengua Española.

4 Nombre que el movimiento artístico de vanguardia La Internacional Situacionista daba a las relaciones societales contemporáneas que desde mediados del siglo XX son definidas por la cultura capitalista occidental. 
metodología proyectual, y en otras, metodologías de investigación-creación pero en este tipo de propuestas metodológicas no es posible reconocer con claridad la injerencia del gusto en tanto factor que condiciona y afecta al proceso y al resultado final. Por tal motivo es importante evidenciar las diferentes formas en las que el gusto, entendido como se ha explicado con anterioridad, incide en algunas fases -si no en todas- del proceso creativo, y finalmente cómo este criterio puede ser entendido de manera independiente para permitir construir un fundamento reflexivo que permita desarrollar una práctica valorativa del arte.

La imbricación del gusto en el ejercicio valorativo de las artes se puede evidenciar al analizar una posible estructura procesual de un proyecto artístico dividido para efectos de este análisis en tres etapas que, aunque se organizan de manera lineal pueden, dependiendo de las características del proyecto, variar en cuanto a su orden o darse de manera simultánea.

En la primera etapa o de estructuración del problema, las distintas operaciones metodológicas dejan de lado un factor determinante e influyente en lo que se refiere a la definición y el análisis de un problema, pues generalmente no se tiene en cuenta al agente, nombre con el que el filósofo Javier Echeverría definiría a personas, máquinas, grupos, instituciones o empresas que realizan alguna acción, y, por lo tanto, se puede decir que crean, que hacen, que ponen en acción el potencial creativo. Los agentes, incluyendo las máquinas y los computadores, tienen unos presupuestos o conocimientos previos que les son indispensables para definir un enfoque, una dirección preconcebida -aunque no desarrollada- del proyecto, y a partir de estos realizar la labor artística; estos constructos teóricos, reflexivos y prácticos les permiten analizar de manera diferente las mismas acciones, condicionando los resultados a las diversas formas de ver problemas axiológicos. El agente es parte del acontecimiento artístico, está inmerso en la acción y la afecta de tal forma, que la distancia valorativa no es posible, pues quien observa ve una acción que en cierta medida es producida por él mismo, convirtiéndose -el agente- en una variable dinámica del problema a definir y de todo el proyecto; así se puede decir que la estructuración de un problema implica un modus y, por lo tanto, un gusto; es así como en determinados momentos históricos y en contextos socioculturales particulares es posible identificar tendencias y focos problemáticos generalizados. Cabe aclarar que no son los problemas en sí mismos los que se pueden enmarcar en una moda particular, sino más bien los enfoques o maneras en las que estos se abordan.

En segunda etapa se debe entender que las operaciones, construcciones o acciones son el resultado del modo en que el agente hace algo, y por lo tanto, esas realizaciones reflejan las maneras particulares de entender el mundo o lo que es lo mismo: los gustos de los agentes. Pero en este punto también se proyecta y diseña, es decir, estas operaciones son al mismo tiempo de orden cognitivo y se dan en el mundo de las ideas. También es posible entender la acción realizada por el agente como un acontecimiento sensible, estético, que se produce como emoción y que afecta al hombre tanto desde las formas perceptibles biológicas, como desde la alteración de sus estados emocionales y espiri- 
tuales. Estas condiciones creativas que permiten la acción del diseño y la relación con lo creado son posibles en tanto quien crea es un ser social que ha desarrollado un lenguaje o unas formas particulares de proyectarse creativamente frente a esa comunidad en la que se inscribe. Por lo tanto, la acción creativa es un acto ya no simplemente subjetivo, sino más bien intersubjetivo, una acción que parte de un modus particular, es decir, de un gusto individual que es construido en dentro de una estructura societal permitiendo la consolidación de un lenguaje o estilo, y que busca, a la vez, afectar el gusto de otros, estando en consonancia con estos o rompiendo la tendencia del momento intentando ser un punto de quiebre y de partida para la consolidación de una nueva moda.

También en esta etapa Bonsiepe incluye una operación evaluativa, y en tal sentido puede decirse que cualquier ejercicio valorativo realizado a un proyecto de diseño que propone cruces continuos de retroacción social, histórica y contextual tendrá que hacerse desde la teoría de los sistemas, de modo que ningún componente puede ser evaluado de manera independiente, sino en relación con todas las variables dentro de la estructura sistémica, de tal forma que la evaluación tenga por objetivo determinar la fiabilidad, la funcionalidad, la robustez, la eficacia, el impacto y las formas de producción, es decir, se ocupe de la revisión de la coherencia entre el proceso y sus efectos; pero estos criterios son insuficientes si no se analiza la pertinencia del diseño en relación con el contexto del que surge y al que afecta.

Hay que aclarar que la evaluación no determina juicios absolutos, sino que es cuestión de grados, de valores relativos de diferente tipo que en conjunto determinan el nivel de importancia o de efectividad de la propuesta. Hay que abandonar el enfoque maximizador, y reemplazarlo por otro de orden axiológico en el que valores básicos, epistémicos, técnicos, económicos, militares, ecológicos, políticos, jurídicos, sociales, religiosos, morales, estéticos, formales y culturales tejen la complejidad evaluativa.

Se puede decir que la evaluación tiene una función validadora; esta es posible gracias al uso de valores intersubjetivos, es decir, compartidos, de modo que independientemente del agente concreto que evalúe el proceso investigativo la valoración realizada sea válida. En este punto es importante afirmar que la evaluación no procura la unificación de criterios, o el logro de un consenso, pues la intersubjetividad se funda en formas de la racionalidad deliberativa y genera disenso; esto no le resta validez a la propuesta valorativa, sino que permite que los juicios realizados se den desde diversidad de gustos y se evite la imposición de un criterio único y subjetivo.

Los procesos de evaluación, al ser realizados por múltiples agentes y cada vez más desde diferentes disciplinas, están sujetos al cambio valorativo pues, "aunque sea en instancias sucesivas de evaluación, los resultados finales han de haber superado diversas cribas axiológicas, que no suelen ser exclusivamente epistémicas o técnicas. Pues bien, cada agente evaluador dentro de este sistema multi-agente promueve sus propios valores, y al hacerlo impregna los subsistemas de valores de los restantes agentes" (Echeverría, 1998, p. 189). Los agentes evaluadores en muchas ocasiones no son personas, sino grupos, comunidades del conocimiento; esto porque en la Contemporaneidad es difícil que 
una sola persona contenga la pluralidad de virtudes requeridas para realizar o evaluar un proceso completo de diseño.

Los criterios de valoración varían constantemente en función de los contextos, las disciplinas, las épocas y las situaciones concretas en las que actúan. Incluso los valores internos o epistémicos surgen y se renuevan en momentos históricos concretos.

En lo que respecta a la tercera etapa, esta puede ser entendida como el resultado de las decisiones tomadas en las dos anteriores. En ella se plantea al mismo tiempo la finalización del proceso proyectual y la materialización de un producto evaluable en relación con la finalidad con la que fue creado o con la usabilidad práctica y sensible, es decir, fáctica y estética. En relación con el gusto puede decirse que el impacto que genere la propuesta y su permanencia en las apetencias estéticas, deseos y afectos de aquellos a quienes se dirige validan o no el diseño nuevamente en una dinámica social e intersubjetiva.

\section{Conclusión}

\section{Un cambio en el juicio de valor del gusto}

Los cambios valorativos se asocian con la noción del bien, pero este concepto tanto en ciencias naturales como en humanas e inclusive en el arte (entendido aquí como parte de las humanidades) tiene un carácter ambivalente; en tal sentido se puede decir que el ser humano no tiene solo la capacidad de crear sino también de destruir; por eso algunos bienes axiológicos, por ejemplo, en la actividad militar, son males para los valores básicos: la victoria, la fuerza, la mayor capacidad destructiva están dispuestas o su acción va en contra de objetivos humanos, generalmente llamados enemigo. En tal sentido los valores deben identificarse con claridad dentro del contexto de la acción misma del diseño y ser juzgados tanto hacia el interior, como hacia el exterior. Desconocer el impacto del diseño por fuera de ellos mismos ha derivado en consecuencias como la reducción de la sostenibilidad del planeta, implicando muchas veces desarrollos que están en contra de la vida.

No solo hay que evaluar los efectos positivos y negativos, por parte de, y para los agentes, sino también para y por los pacientes, es decir, para los objetos o personas sobre los cuales se ejerce la acción proyectiva del diseño.

Como criterio general, se puede decir que será mejor aquel diseño que tenga la capacidad de integrar diversos sistemas de valores y gustos, a veces opuestos y en conflicto, de modo que la satisfacción de todos y cada uno de ellos sea exigible, aunque sea en un cierto grado. Esto no quiere decir que exista algo así como un diseño bueno que aporta ventajas y uno malo que solo produce perjuicios, sino que desde el comienzo del proceso creativo, es decir, mediante el cual se proyectan las ideas se pueda comprender la ambivalencia, la complejidad que vive en el centro mismo del conocimiento en el campo del diseño.

Las múltiples aproximaciones permiten dirigir la discusión a consideraciones materiales que son fundamentales para el ejercicio y la producción de las valoraciones y ejercicios pedagógicos dentro del diseño como lo ha planteado Bourdieu (Bourdieu, 1995), desde la indagación del perfil profesional del intelectual-escritor como capital simbólico, hasta los 
criterios de formalización de las mismas producciones. Asimismo, se puede considerar la elección del intelectual de vincular su tarea en los campos de sentido, propuestos por el ámbito institucional y académico; o también la intención de permanecer distanciado de los medios de visibilidad modernos y racionalistas, caso que le posibilita la reflexión sobre la propia disciplina y la indagación de sus propios principios de legitimidad, y que lo ubicaría en una situación de autonomía.

\section{Bibliografía}

Addison, J. (1991). Los placeres de la imaginación y otros ensayos de The Spectator. Madrid: Visor.

Arnheim, R. (1965). Art and Visual Perception. Berkeley and Los Ángeles: University of California Press

Arnheim, R. (1995): Arte y percepción visual: psicología del ojo creador. Madrod: Alianza Forma.

Bonsiepe, G. (1993). Del objeto a la interface. Buenos Aires: Infinito.

Bonsiepe, G. (1993). Las 7 columnas del diseño. México: Universidad Autónoma Metropolitana Azcapotzalco.

Echeverría, J. (2002). Ciencia y valores. Madrid: Destino.

Echeverría, J. (1998) Filosofía de la ciencia. Madrid: Akal.

Gadamer, H. (2000). Verdad y método, Madrid: Ediciones Sígueme.

Gerard, A. (1992). El ensayo sobre el gusto. Valencia: Universidad de Valencia.

Hume. D. (1989). Sobre la norma del gusto. Barcelona: Ediciones Península.

Hutcheson, F. (1992). Una investigación sobre el origen de nuestra idea de belleza. Madrid: Tecnos.

Kant, E. (1995). Crítica del juicio. Madrid: Espasa Calpe.

Kant, E. (1990). Observaciones acerca del sentimiento de lo bello y lo sublime. Madrid: Alianza.

Nó, J. (2000): "Imagen impuesta, imagen negociada". Trípodos: llenguatge-pensament-comunicació. Número extra Comunicació $i$ Realitat, págs. 413-422.

Osgood, CH. E. et al. (1976): La medida del significado. Madrid: Gredos.

Schiller, F. (1990). Cartas sobre la educación estética del hombre. Barcelona: Anthropos. 